\title{
Anaphylactoid reaction to immunoadsorptive membrane in a patient with myasthenia gravis
}

\author{
Yoshihiko Kanno • Shigeaki Suzuki • \\ Tadashi Yoshida $\cdot$ Yugaku Date $\cdot$ Norihiro Suzuki \\ Matsuhiko Hayashi
}

Received: 20 September 2011/ Accepted: 10 November 2011/Published online: 28 December 2011

(c) Japanese Society of Nephrology 2011

\begin{abstract}
Extracorporeal adsorption therapy has been carried out clinically in cases of myasthenia gravis (MG) since the 1980s without any major complications. Despite a lack of clinical evidence, it has been accepted as a potential therapy for steroid-resistant patients in many institutes. Recently, we experienced a case of MG with an anaphylactoid reaction during extracorporeal immunoadsorption therapy. A 64 year old women with MG showed an anaphylactoid reaction immediately after the initiation of adsorption therapy using a polyvinylalcohol gel column. It is therefore important to bear in mind that extracorporeal adsorption therapy could trigger an anaphylactoid reaction, which may induce a MG crisis.
\end{abstract}

Keywords Crisis - Myasthenia gravis - TR350 - Shock

\section{Background}

Myasthenia gravis (MG) is an autoimmune neuromuscular disease leading to fluctuating muscle weakness and fatigability. It is an autoimmune disorder in which weakness is caused by circulating antibodies that block acetylcholine receptors at the postsynaptic neuromuscular junction [1]. Extracorporeal adsorption therapy was introduced clinically for cases of MG in the 1980s, and is now accepted

Y. Kanno $(\bowtie) \cdot$ T. Yoshida $\cdot$ M. Hayashi

Apheresis and Dialysis Center, School of Medicine,

Keio University, 35 Shinanomachi, Shinjuku,

Tokyo 160-8582, Japan

e-mail: kannoyh@a3.keio.jp

S. Suzuki · Y. Date $\cdot$ N. Suzuki

Department of Neurology, School of Medicine,

Keio University, Tokyo, Japan as a potential therapy for steroid-resistant patient [2], despite insufficient evidence [3]. The therapy is considered relatively safe because adverse effects of it have only seldom been reported, except for a mild reduction in blood pressure. Recently, we experienced a patient with MG who had an anaphylactoid reaction during extracorporeal immunoadsorption therapy.

\section{Case report}

A 64 year old woman with thymoma-associated MG was admitted to our hospital due to worsening bulbar symptoms. She first noticed right-sided ptosis 5 months before admission, and received a diagnosis of $\mathrm{MG}$ based on clinical, electrophysiological, and immunological findings. In addition to anti-acetylcholine receptor antibody, she also had anti-Kv1.4 antibody, a serological marker for thymoma and severe MG [4]. She had received thymomectomy (Masaoka grade I and WHO type AB) 2 months before admission. She had no previous history of drug or food allergies, and had not been administered angiotensinconverting enzyme inhibitor for any reason. Her MG had gradually worsened, and she required pyridostigmine $120 \mathrm{mg} /$ day and prednisolone $20 \mathrm{mg}$ /day on admission (Fig. 1). Extracorporeal immunoadsorptive therapy was ordered: an Immusorba TR350 (Asahi Medical, Tokyo, Japan) was used as the adsorptive column, and a Plasmaflo OP-05W (Asahi Medical) was incorporated into the circuit as a plasma separator. In the procedure, the intention was to withdraw the blood at a rate of $100 \mathrm{ml} / \mathrm{min}$ through a catheter in the femoral vein until $2,000 \mathrm{ml}(36 \mathrm{ml} / \mathrm{kg})$ of plasma had been treated by the column. Anticoagulation was achieved by administering 1,500 $\mathrm{U}$ of heparin. However, ten minutes after the start of the session, she claimed 


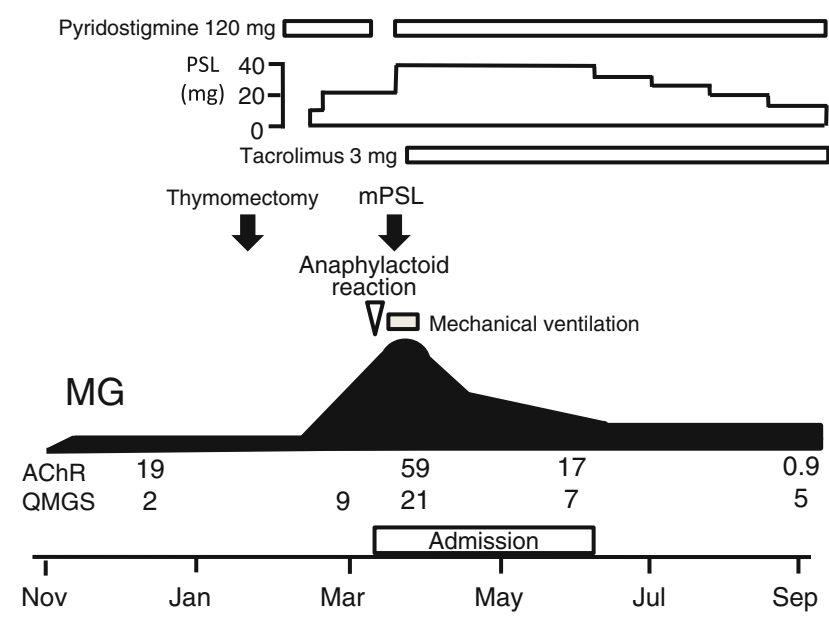

Fig. 1 Clinical course of our patient. PSL prednisolone, mPSL methylprednisolone, $M G$ myasthenia gravis, $A C h R$ anti-acetylcholine receptor antibody, $Q M G S$ quantitative myasthenia gravis score

chest discomfort. Medical doctors observed that she had a flushed face, systemic eczyma, and tachypnea. As her oxygen saturation decreased from 99 to $83 \%$, a medical doctor decided to stop the session and follow emergency procedures. At the end of the study, her white blood cell count had increased from 7,500 to 28,400 per microliter. Incubation was carried out and she was treated generally in the ICU. She recovered from the crisis after receiving steroid pulse therapy for a few days.

\section{Discussion}

A mild reduction in blood pressure is the main reported adverse effect of extracorporeal adsorptive therapy using Immusorba TR350, and this occurred $1 \mathrm{~h}$ after the start of therapy in almost all cases [5]. In our case, all symptoms specific for anaphylactoid reaction were found immediately after the start of therapy. The TR350 column contains a polyvinylalcohol gel to which tryptophan is covalently attached as a ligand, and achieves its adsorptive capacity through hydrophobic forces and the anionic charge of tryptophan [6]. OP-05W is made from polyethylene through gamma ray sterilization. Polyethylene is the most widely used plastic of all (e.g., in plastic bags), and there is no report of it causing anaphylaxis either. Anaphylactoid reactions caused by the sterilizer, plastic tube, or its plasticizer diethylhexyl phthalate (DEHP) have also not been reported. Heparin could act as an allergen in an anaphylactoid reaction [7]. The administration of pyridostigmine might be a risk factor for cholinergic crisis during extracorporeal circulation. However, the presence of muscarinic (abdominal cramps, diarrhea, lacrimation, meiosis) or nicotinic (fasciculations, muscle cramps) signs was not observed throughout the clinical course. Heart rate was 90 beats/min at the start of the session, and it increased to 117 beats/min at the end of session. $120 \mathrm{mg}$ of pyridostigmine seemed to be an insufficient dose to cause cholinergic crisis. Unfortunately, we did not measure the peripheral eosinophil count or the serum concentration of IgE. Although no antigen for this reaction was found, and allergic markers were not measured, we considered that our patient experienced an anaphylactoid reaction to TR350 clinically. An anaphylactoid reaction to TR350 was also reported in 2003 for 1 of 9 patients with pemphigus [8]. In our hospital, extracorporeal adsorptive therapy using TR350 was first used in 1998 for patients with MG. By 2011, 30 patients had received extracorporeal adsorptive therapy, and no patients had shown any major complication with the therapy. In contrast, plasma exchange was carried out for $6 \mathrm{MG}$ patients who were negative for antiacetylcholine receptor antibody, but 2 patients were positive for anti-muscle-specific tyrosine kinase. We have never experienced major complications with extracorporeal adsorptive therapy with any of our patients. Also, in Japan, despite the fact that 50,000 TR350 columns have been used for hemoperfusion therapy since 1989, no major complication arising from their use has been reported (personal communication). Thus, extracorporeal adsorption therapy is considered to be relatively safe, but it is important to bear in mind that it could possibly act as a trigger for an anaphylactoid reaction leading to a lethal MG crisis. Epidemiological research should be carried out concerning this therapy.

Acknowledgments The author thanks Mr. Eiji Kubiura, Asahi Kasei Kuraray Medical Co., Ltd., for providing product information on TR350.

\section{References}

1. Angelini C. Diagnosis and management of autoimmune myasthenia gravis. Clin Drug Investig. 2011;31:1-14.

2. Psaridi-Linardaki L, Mamalaki A, Tzartos SJ. Future therapeutic strategies in autoimmune myasthenia gravis. Ann N Y Acad Sci. 2003;998:539-48.

3. Cortese I, Chaudhry V, So YT, Cantor F, Cornblath DR, Rae-Grant A. Evidence-based guideline update: plasmapheresis in neurologic disorders: report of the Therapeutics and Technology Assessment Subcommittee of the American Academy of Neurology. Neurology. 2011;76:294-300.

4. Suzuki S, Utsugisawa K, Nagane Y, Satoh T, Terayama Y, Suzuki $\mathrm{N}$, et al. Classification of myasthenia gravis based on autoantibody status. Arch Neurol. 2007;64:1121-4.

5. Grob D, Simpson D, Mitsumoto H, Hoch B, Mokhtarian F, Bender A, et al. Treatment of myasthenia gravis by immunoadsorption of plasma. Neurology. 1995;45:338-44. 
6. Yuki N. Tryptophan-immobilized column adsorbs immunoglobulin G anti-GQ1b antibody from Fisher's syndrome: a new approach to treatment. Neurology. 1996;46:1644-51.

7. Warkentin TE, Greinacher A. Heparin-induced anaphylactic and anaphylactoid reactions: two distinct but overlapping syndromes. Expert Opin Drug Saf. 2009;8:129-44.
8. Luftl M, Stauber A, Mainka A, Klingel R, Schuler G, Hertl M. Successful removal of pathogenic autoantibodies in pemphigus by immunoadsorption with a tryptophan-linked polyvinylalcohol adsorber. Br J Dermatol. 2003;149:598-605. 